\title{
Does the extracorporeal circulation worsen anemia in hemodialysis patients? Investigation with advanced microscopes of red blood cells drawn at the beginning and end of dialysis
}

\author{
This article was published in the following Dove Press journal: \\ International Journal of Nanomedicine \\ 10 October 2013 \\ Number of times this article has been viewed
}

\author{
Dimosthenis Stamopoulos' \\ Nerantzoula Bakirtzi ${ }^{2,3}$ \\ Efthymios Manios' \\ Eirini Grapsa ${ }^{4}$ \\ 'Institute of Advanced Materials, \\ Physicochemical Processes, \\ Nanotechnology and Microsystems, \\ National Center for Scientific \\ Research 'Demokritos,' Athens, \\ Greece; 'Department of Nephrology, \\ Hospital 'G. Gennimatas,' Athens, \\ Greece; ${ }^{3}$ Renal Unit, Hospital \\ 'Alexandra,' Athens, Greece; ${ }^{4}$ Renal \\ Unit, Hospital 'Aretaieion,' Athens, \\ Greece
}

\begin{abstract}
Background: In hemodialysis (HD) patients, anemia relates to three main factors: insufficient production of erythropoietin; impaired management of iron; and decreased lifespan of red blood cells (RBCs). The third factor can relate to structural deterioration of RBCs due to extrinsic (extracorporeal circuit; biochemical activation and/or mechanical stress during dialysis) and intrinsic (uremic milieu; biochemical interference of the RBC membrane constituents with toxins) mechanisms. Herein, we evaluate information accessed with advanced imaging techniques at the cellular level.
\end{abstract}

Methods: Atomic force and scanning electron microscopes were employed to survey intact RBCs (iRBCs) of seven HD patients in comparison to seven healthy donors. The extrinsic factor was investigated by contrasting pre- and post-HD samples. The intrinsic environment was investigated by comparing the microscopy data with the clinical ones.

Results: The iRBC membranes of the enrolled HD patients were overpopulated with orificelike (high incidence; typical size within 100-1,000 nm) and crevice-like (low incidence; typical size within 500-4,000 nm) defects that exhibited a statistically significant $(P<0.05)$ relative increase $(+55 \%$ and $+350 \%$, respectively) in respect to healthy donors. The relative variation of the orifice and crevice indices (mean population of orifices and crevices per top membrane surface) between pre- and post-HD was not statistically significant $(-3.3 \%$ and $+4.5 \%$, respectively). The orifice index correlates with the concentrations of urea, calcium, and phosphorus, but not, however, with that of creatinine.

Conclusion: Extracorporeal circulation is not detrimental to the structural integrity of RBC membranes. Uremic milieu is a candidate cause of RBC membrane deterioration, which possibly worsens anemia.

Keywords: hemodialysis, anemia, red blood cells, atomic force microscopy, scanning electron microscopy

\section{Introduction}

In hemodialysis (HD) patients, anemia is caused and/or worsened by three main factors: (1) insufficient production of endogenous erythropoietin (EPO) that results in decreased production of red blood cells (RBCs) by the bone marrow; (2) impaired management of iron, due to nutritional restrictions and suppressed iron absorption by the gastrointestinal tract, that influences RBC maturation; and (3) decreased lifespan of RBCs (up to 70\%), which, although observed in virtually all HD patients, is still of unknown etiology. ${ }^{1-7}$ 
In this study we focus on the third factor mentioned above, that is, the decreased lifespan of RBCs, since all studied HD patients were adequately supplemented with exogenous EPO and iron agents. The possible mechanisms motivating and/or promoting the decreased lifespan of RBCs can be categorized as extrinsic (extracorporeal circuit) or intrinsic (uremic milieu). The extrinsic mechanisms relate to the extracorporeal circulation of the HD patient's blood during dialysis, since this can induce mechanical stress, ${ }^{8,9}$ biochemical activation, ${ }^{10,11}$ and membrane compositional modifications ${ }^{12-15}$ to the RBCs. Intrinsic mechanisms refer to the interference of the biochemically crowded landscape of the RBC membranes (receptors, transport channels, etc) with the complex environment of uremic toxins. ${ }^{16}$ Revealing the mechanisms that motivate and/or promote the reduction of RBCs lifespan is an issue of both basic research and clinical importance, since once the underlying cause is revealed, new preventative strategies can be found by which to restore RBCs lifespan back to normal levels, thus establishing more rational limits to the therapeutic use of EPO and iron agents.

For the purposes of this study, we employed atomic force microscopy (AFM) and scanning electron microscopy (SEM), two powerful techniques that can reveal precise information on both the overall morphology of cells at the micrometer $\left(1 \mu \mathrm{m}=10^{-6} \mathrm{~m}\right)$ level and the membrane characteristics of cells at the nanometer $\left(1 \mathrm{~nm}=10^{-9} \mathrm{~m}\right)$ level. ${ }^{7,17-25}$ With AFM and SEM, we accessed thorough structural information from the membranes of intact RBCs (iRBCs) of HD patients, drawn both pre- and post-HD. A comparison to healthy donors was also performed.

\section{Subjects and methods Study design}

This study was conducted in a time frame of almost 2 years, during which we executed repeated iRBCs examinations, from seven HD patients and seven healthy donors, with both AFM and SEM.

\section{HD patients}

All seven HD patients enrolled in this study, after informed consent and with the approval of the ethics review board of Hospital 'Alexandra', Athens, Greece, underwent thriceweekly 4-hour dialysis sessions at the 'Alexandra' Hospital's renal unit. Exclusion criteria were based on blood transfusion in a time window of less than 3 months, initiation of HD in a time window of less than 3 months, current history of smoking, and use of medications of hemolytic action. For the specific patients studied in this work, the cause of end-stage renal disease refers to amyloidosis (two patients), glomerulonephritis (one patient), polycystic kidney disease (two patients), Wegener's granulomatosis (one patient), and diabetes mellitus (one patient). All biochemical and hematological data were obtained by means of standard clinical practice methods.

\section{Healthy donors}

The seven healthy donors were chosen by the authors and colleagues, after informed consent was obtained. Matching the sex and age parameters was a primary registration criterion for the healthy donors. Healthy donors were not subjected to any medication during the study and had no record of any chronic/current disease or hematological disorder.

\section{Materials}

Standard anticoagulant-containing diaminoethanetetraacetic acid (EDTA) Vacutainer test tubes (BD, Franklin Lakes, NJ, USA) were used for temporary storage of blood samples. Standard glass microscope slides (Corning Incorporated, Corning, NY, USA) were used for the preparation of iRBC films for AFM imaging. Standard silicon wafers (p-type, B-doped, $<100>$ orientation; SIEGERT WAFER GmbH, Aachen, Germany) were used for the preparation of iRBC films for SEM imaging.

\section{Methods}

\section{Dialysis details}

The HD patients underwent dialysis with various membranes: polysulfone (Bio-140; Dialife SA, Taverne, Switzerland); hemophan (GFS 16 plus; Gambro, Hechingen, Germany); polyester polymer alloy (FDX-210GW; Nikkiso, Tokyo, Japan); and ethylene vinyl alcohol copolymer (KF-201-m; Kawasumi Laboratories Inc, Tokyo, Japan). The employed dialyzate was standard bicarbonate $(\mathrm{Na} 140 \mathrm{mmol} / \mathrm{L}$, $\mathrm{HCO}_{3} 35 \mathrm{mmol} / \mathrm{L}, \mathrm{K} 2.5 \mathrm{mmol} / \mathrm{L}, \mathrm{Ca} 1.75 \mathrm{mmol} / \mathrm{L}, \mathrm{Mg}$ $0.75 \mathrm{mmol} / \mathrm{L}$ ) of high bacteriological quality water at a flow rate of $500 \mathrm{~mL} /$ minute.

\section{Blood collection and preparation of iRBC films}

Both pre- and post-HD, for all HD patients, whole blood was drawn from the arterial line of the extracorporeal circuit 5 minutes after the initiation of the process and before the end of the dialysis session, respectively. For the healthy donors, whole blood was drawn by venipuncture. All blood samples were deposited in standard anticoagulant-containing (EDTA) test tubes. According to our experimental protocol, blood was always used fresh within 2 hours after collection (in some 
cases, samples were temporarily stored at $\mathrm{T}=4^{\circ} \mathrm{C}$ and used within 48 hours; the results were unaltered). Blood samples were subjected to the minimum possible processing, since Stamopoulos et al, in a recent study, ${ }^{19}$ showed that media considered to be perfectly inert (such as saline, phosphatebuffered saline, etc) influence blood cells, modifying the original morphological information of their membrane, at least at the nanometer level. Thus, in this study, we focused on iRBCs, that is, RBCs in whole plasma, by keeping additional processing to a minimum. Specifically, dilutions with a final hematocrit value of $5 \%-10 \%$ that were needed for the preparation of blood films were prepared by means of autologous plasma.

After dilution, the iRBC films (smears) were deposited onto two kinds of substrate, depending on the microscope technique used for imaging. For AFM imaging, the employed substrates were standard microscope glass slides (Corning Incorporated) that were carefully cleaned with ethanol, without any extra processing. For SEM imaging, the respective substrates were standard silicon pieces, precoated with an ultraclean and relatively thick $(100 \mathrm{~nm})$ niobium layer by means of a professional sputtering unit (Edwards 306A; Edwards, Sanborn, NY, USA) under high vacuum ( $10^{-8}$ Torr). The niobium buffer layer was necessary to make the silicon substrate conductive without loss of its original surface characteristics (ultraclean surface of roughness below $1 \mathrm{~nm}$ ).

For the preparation of the iRBC films (smears), the glass slide/silicon substrate was placed onto an electronically controlled platform (Headway Research INC MOTOR CONTROL-101) that starts to rotate at a prefixed speed $(2,000$ $3,500 \mathrm{rpm}$ ) and duration (5-15 seconds) after the deposition of a small amount of blood $(50-100 \mu \mathrm{L}$ is adequate to produce a film with dimensions in the range of $\mathrm{cm}$ ). The centrifugal force exerted onto the blood drop produces single-layered and uniform films (smears) of well-separated, appropriately closely packed, iRBCs. Thus, these iRBC films are in "dry" form; during the deposition process with the spinner, the iRBCs are dried almost instantly (within a few seconds), since "excessive" plasma is rejected. We stress that, not only during but also after their preparation, the iRBC films were left intact without any fixation except for the deposition of a thin gold overlay that was a necessary requirement for the investigation with SEM (see below).

\section{AFM and SEM techniques}

AFM data were acquired on iRBC films, deposited on glass slides and left in dry form as discussed above, by means of a scanning probe microscope (Solver PRO; NT-MDT Co,
Moscow, Russia) with a $100 \times 100 \times 5 \mu \mathrm{m}^{3} \mathrm{XYZ}$ scanner hosted on an active vibration isolation table (MOD-1M plus; Halcyonics GmbH, Goettingen, Germany). Our measurements were performed in the noncontact scanning mode with NCH cantilevers that end with silicon nitride tips (Nano and More $\mathrm{GmbH}$, Wetzlar, Germany) and have the nominal parameters, spring constant $=42 \mathrm{Nm}^{-1}$ and resonance frequency $=320 \mathrm{kHz}$. The optimum imaging results were obtained within the following scanning parameters: line frequency $=1.5-4 \mathrm{~Hz}$, area $=0.5 \times 0.5-50 \times 50 \mu \mathrm{m}^{2}$, and lines per image $=256-512$.

SEM investigations of the iRBC films, deposited on niobium-precoated silicon substrates and left in dry form as discussed above, were performed by means of an Inspect microscope (FEI, Hillsboro, OR, USA) working with W (tungsten) filament. Regarding the sample preparation stage, the niobium-precoated silicon substrate hosting the iRBC film was glued with silver paste onto conventional pin stubs, after which a thin overlay $(5-10 \mathrm{~nm})$ of gold was deposited under medium vacuum ( $10^{-1}$ Torr) by means of a typical sputtering unit (SEM coating unit [E5100; Quorum Technologies Ltd, East Sussex, UK]). The role of the gold overlay was two-fold: to make the sample conductive and to adequately protect it from possible cumulative heating and ultimate evaporation from the electronic beam. We stress that the thickness of the gold overlay should be carefully controlled to 5-10 nm, as features of the RBC membrane can be obscured when a relatively thick gold overlay is used. During SEM imaging, we employed an acceleration voltage of $20-30 \mathrm{kV}$, a working distance of 8-12 mm, and spot size of 2-4.

\section{Statistical analysis}

Statistical analysis of the obtained results was performed by means of the Origin software package (Origin 8, OriginLab Corporation, Northampton, MA, USA), and standard Student's $t$-test. Data are presented as mean value \pm standard deviation (SD). Differences were considered not significant at $P>0.05$, statistically significant at $P<0.05$, very significant at $P<0.01$, and extremely significant at $P<0.001$.

\section{Results}

Blood samples were investigated with AFM both at the beginning (pre-HD [Figure 1A-D]) and the end (post-HD [Figure 1E-H]) of dialysis. Three-dimensional renderings of the whole cell (pre-HD [Figure 1A] and post-HD [Figure 1E]) and most important two-dimensional presentations of specific subareas of the RBC membranes (pre-HD [Figure 1B] and post-HD [Figure 1F]) were accessed with AFM. These images 

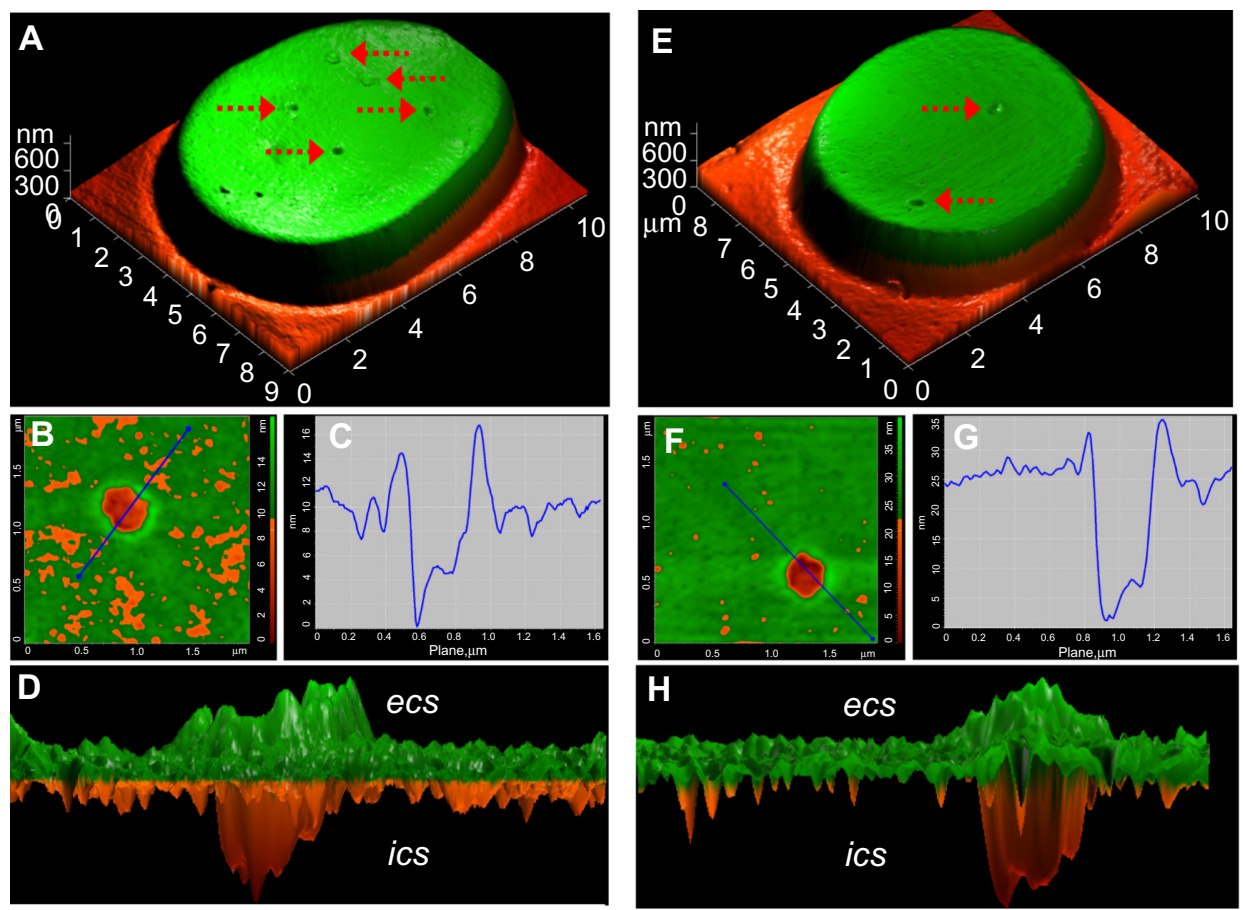

Figure I iRBC investigation with AFM.

Notes: Representative AFM data for (A-D) pre-HD and (E-H) post-HD. (A and E) Three-dimensional renderings of whole iRBCs. (B and F) Two-dimensional presentation of part of the iRBC membrane. (C and $\mathbf{G}) \mathbf{Z}$ profile of the respective line scans shown in $(\mathbf{B})$ and $(\mathbf{F})$. ( $\mathbf{D}$ and $\mathbf{H})$ Three-dimensional, side-view renderings of $(\mathbf{B})$ and $(\mathbf{F})$. Orifices are shown with dotted arrows.

Abbreviations: AFM, atomic force microscopy; ecs, extracellular space; HD, hemodialysis; ics, intracellular space; iRBC, intact red blood cell.

show that the RBC membrane exhibits local defects of circular form (Figure 1A and E), termed "orifices" for the rest of this work. ${ }^{7,19}$ These defects were also recorded in the RBCs of the healthy donors. ${ }^{7,19}$ An advantage of the AFM technique originates from the fact that obtained information of nanometer resolution comes in digital form. Thus, it can be processed with the relevant software so that all the desired dimensions of the observed characteristics can be estimated with great accuracy by using the $\mathrm{Z}$ profile option (pre-HD [Figure 1C] and post-HD [Figure 1G]) of a specific line scan (pre-HD [Figure 1B] and post-HD [Figure 1F]). From these data we can get detailed information on the dimensions of the orifices. Summarizing all the data, we observed that the typical diameters and depths of the orifices ranged from $100-1,000 \mathrm{~nm}$ and 5-100 nm, respectively. The digital form of the AFM data enabled us to reveal the penetration of the membrane defects from the extracellular space to the intracellular space with three-dimensional (side-view) renderings (pre-HD [Figure 1D] and post-HD [Figure 1H]).

Except for the orifices that were circular defects, we observed linear defects of the RBC membranes, termed "crevices." Interestingly, the crevices are quite rare features exhibiting an order of magnitude lower relative incidence when compared to the orifices (see below). Extending the previous work by Stamopoulos et al, ${ }^{7}$ here we employed SEM (in addition to AFM) to validate the observation of these RBC membrane defects with another technique that is minimally invasive (though not entirely noninvasive like AFM). The investigation of blood smears with SEM (Figure 2A) revealed the orifices and crevices of the RBC membranes as well (Figure $2 \mathrm{~B}$ and $\mathrm{C}$ ). With high magnification, the exact dimensions of these defects could be estimated with accuracy, while the fine gold particles deposited to make the sample conductive could also be seen (Figure 2D). We stress that the thickness of the gold overlay should be carefully controlled between 5-10 nm, otherwise the membrane defects are obscured.

The microscopy results on the orifice $\left(\mathrm{R}_{\text {or }}^{\text {Pre/Pst }}\right)$ and crevice $\left(\mathrm{R}_{\mathrm{cr}}{ }^{\text {Pre/Post }}\right.$ ) indices (mean values of the respective defects observed per top surface of the iRBC membrane) for the preand post-HD iRBCs are quantitatively summarized in Table 1. These data demonstrate that the orifice and crevice indices are unaltered between the pre- and post-HD samples (2.66 vs 2.55 and 0.17 vs 0.19 , respectively [no statistically significant variation, $-4 \%$ and $+12 \%$, respectively]). The same behavior is evidenced by the mean value indices $\left(<\mathrm{R}_{\text {or }}^{\text {Pre/Post }}> \pm \mathrm{SD}\right.$ and $<\mathrm{R}_{\text {cr }}$ PrePost $> \pm \mathrm{SD}$ ), which summarize the statistics among the $\mathrm{N}^{\text {Pre/Post }}$ AFM experiments performed in the pre- and post-HD stages. For the healthy donors, the orifice and crevice indices 

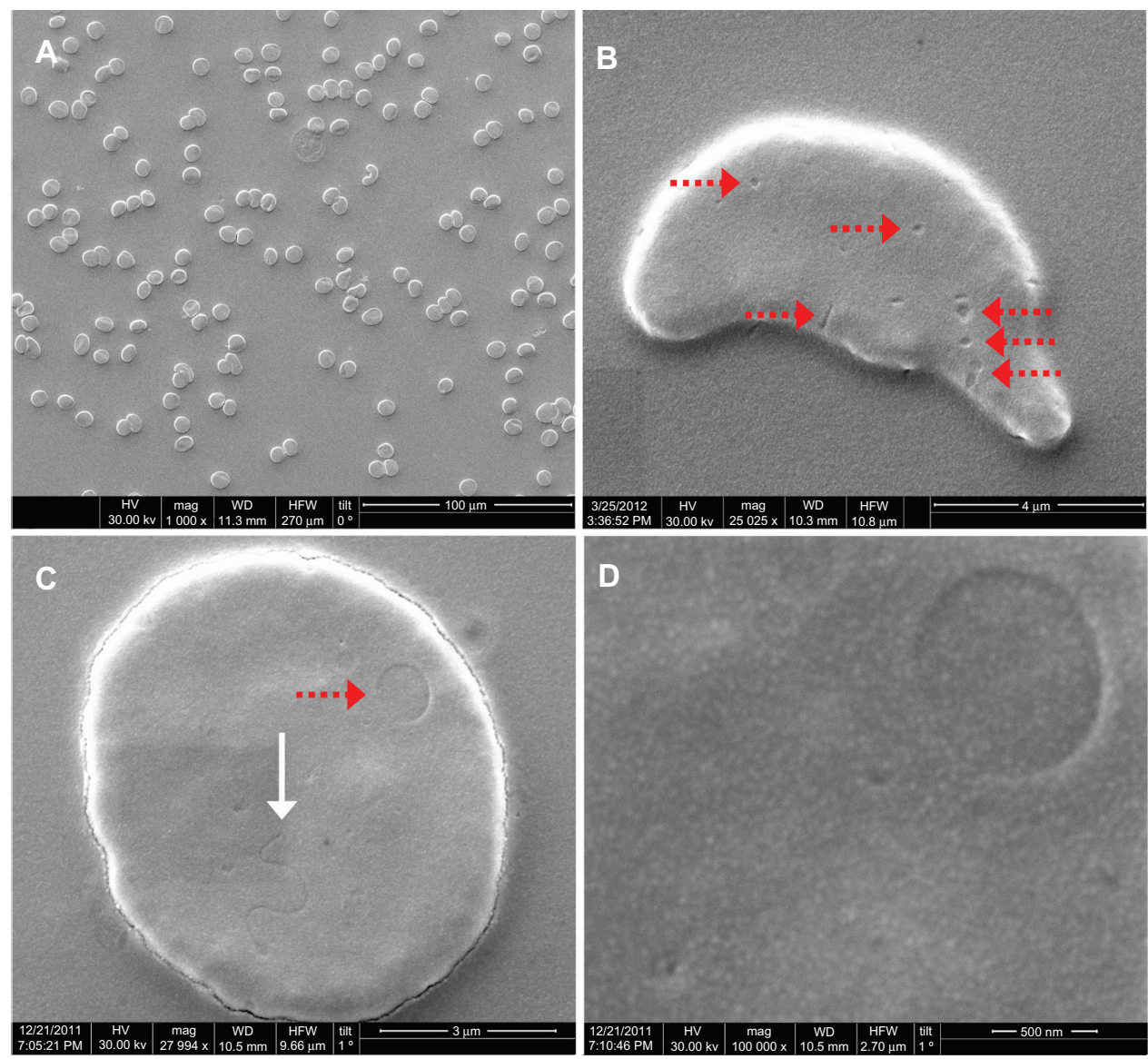

Figure 2 iRBC investigation with SEM.

Notes: Representative SEM data for (A) an extended area of the smear $(\times I, 000)$; (B) whole iRBC that exhibits both numerous orifices and overall shape deformation $(\times 25,000)$; (C) whole iRBC that exhibits an orifice and a crevice $(\times 28,000)$; and $(\mathbf{D})$, focusing on part of the iRBC membrane that exhibits the circular defect $(\times 100,000)$. Orifices and crevices of the RBC membranes are shown with horizontal dotted and vertical solid arrows, respectively.

Abbreviations: iRBC, intact red blood cell; RBC, red blood cell; SEM, scanning electron microscopy.

were $1.67 \pm 0.43$ and $0.05 \pm 0.06$, respectively. The relative percentage increase observed in the HD patients in respect to the healthy donors was approximately $55 \%$ for the orifices and $350 \%$ for the crevices.

To investigate if the orifice index correlated with the concentration of basic uremic markers (urea, creatinine, calcium, and phosphorous), we divided the HD patients into two subgroups, of low and high uremic marker concentration, using the healthy donors as reference group. The results are shown in Figure 3.

\section{Discussion}

The orifices and crevices of the iRBC membranes were observed in both the HD patients and healthy donors,

Table I Orifice and crevice indices, relative variation, and summary of statistics (mean value \pm SD) for the pre and post-HD iRBCs

\begin{tabular}{|c|c|c|c|c|c|}
\hline & iRBCs studied & $\mathbf{R}_{\text {or }}^{\text {Pre/Post* }}$ & $\mathbf{R}_{\text {cr }}^{\text {Pre/Post* }}$ & $<\mathbf{R}_{\text {or }}{ }^{\text {Pre/Post }}> \pm \mathbf{S D}\left(\mathbf{N}^{\text {Pre/Post }}\right)^{\ddagger, \S}$ & $<\mathbf{R}_{\text {cr }}{ }^{\text {Pre/Post }}> \pm \mathbf{S D}\left(\mathbf{N}^{\text {Pre/Post }}\right)^{\ddagger, \S}$ \\
\hline \multicolumn{6}{|l|}{ HD patients } \\
\hline Pre-HD & 185 & 2.66 & 0.17 & $2.68 \pm 0.58(12)$ & $0.22 \pm 0.17(12)$ \\
\hline Post-HD & 172 & 2.55 & 0.19 & $2.59 \pm 0.60(9)$ & $0.23 \pm 0.09(9)$ \\
\hline Variation (\%) ${ }^{\dagger}$ & +7 & -4 & +12 & $-3.3 \pm 2.1$ & $+4.5 \pm 2.8$ \\
\hline
\end{tabular}

Notes: $* R^{\text {Prelpost }}$ and $R_{c r}^{\text {Prelpost }}$ refer to the mean values of 'orifices' and 'crevices' per iRBC top surface for the pre-HD (Pre) and post-HD (Post) populations of iRBCs as if a single experiment was conducted in each case; 'variation (\%) of $R_{o r}{ }^{\text {Pre/Post }}$ and $R_{c r}$ Pre/Post refer to the relative percentage variation of the mean values obtained for the post$H D$ samples in respect to the pre-HD ones and is defined through the relation $\left[\left(R_{\text {or } / c r}^{\text {Post }} R_{\text {or/cr }}^{\text {Pre }}\right) / R_{\text {or/cr }}^{\text {Pre }}\right] 100 \%$; $\neq<R_{\text {or }}^{\text {Prelpost }}>$ and $<R_{\text {cr }}^{\text {Prespost }}>$ are defined by $<R_{\text {or/cr }}^{\text {Prelpost }}>=$

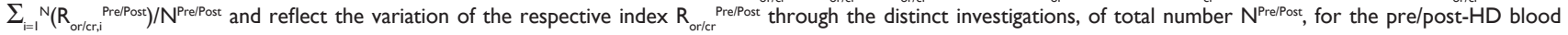
samples of the HD patients; ' with ( $\mathrm{N}^{\text {Pre/Post})}$ ) by the 'mean value \pm SD' at the two last columns we denote the total runs of AFM investigations for the pre-HD and post-HD samples of the HD patients.

Abbreviations: AFM, atomic force microscopy; HD, hemodialysis; iRBCs, intact red blood cells; $R_{c r}$, crevice index; $R_{o r}$, orifice index; $S D$, standard deviation. 

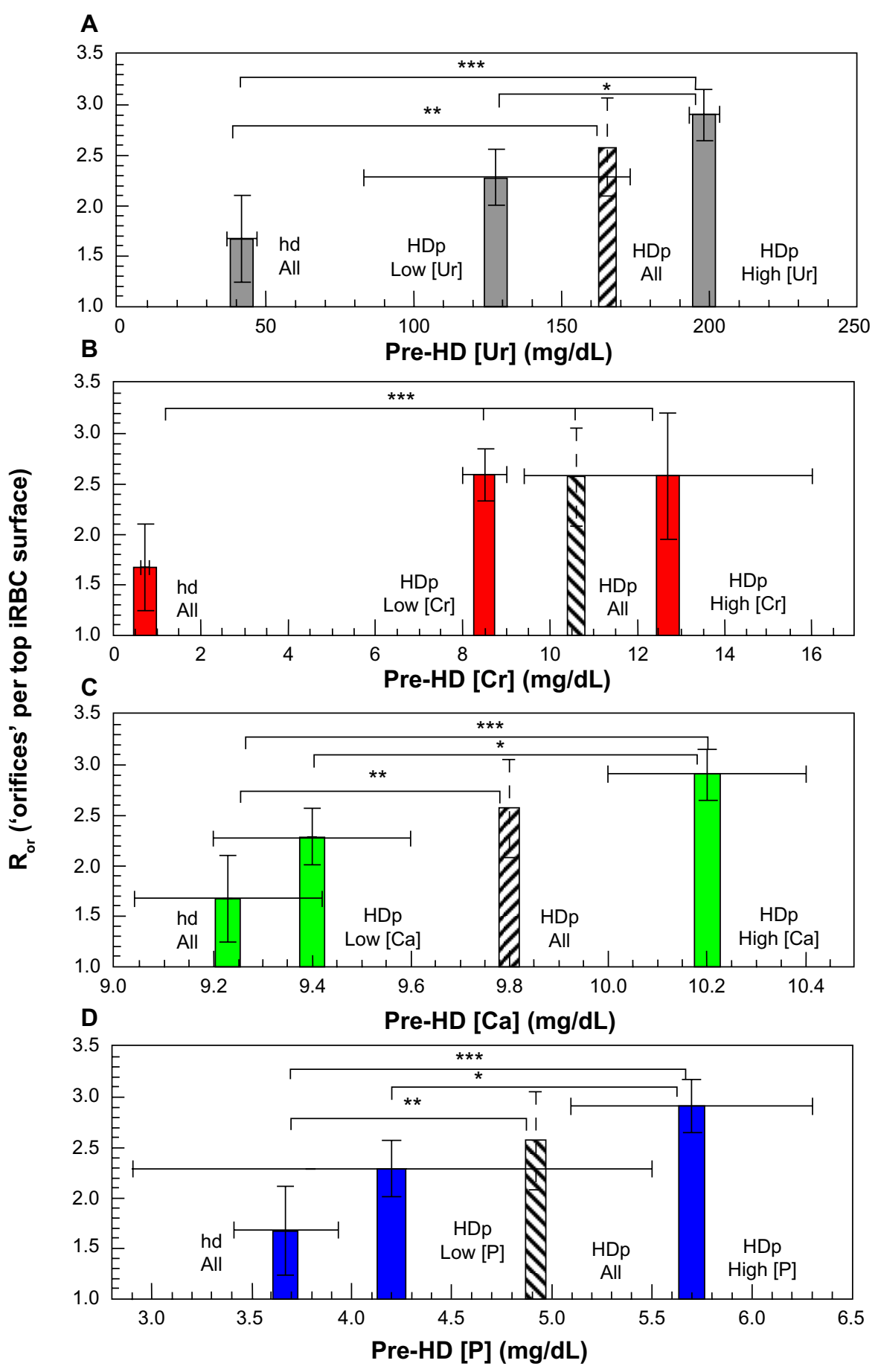

Figure 3 Correlation of $\mathrm{R}_{\text {or }}$ with the concentration of basic uremic markers.

Notes: Dependence of $\mathrm{R}_{\text {or }}$ with $(\mathbf{A})$ pre-HD Ur; (B) pre-HD Cr, (C) pre-HD Ca; and (D) pre-HD P, comparison was between all different pairs: the two subgroups of HD patients, of low and high uremic marker concentration, and the group of healthy donors. The data for the complete group of HD patients are also shown for clarity (hatched columns). Statistically significant differences observed between (sub)groups are denoted in all cases. $* P<0.05$ (significant); $* * P<0.0$ I (very significant); $* * * P<$ 0.001 (extremely significant).

Abbreviations: Ca, calcium; Cr, creatinine; hd, healthy donors; HD, hemodialysis; HDp, HD patients; iRBC, intact red blood cell; $\mathrm{P}$, phosphorous; $\mathrm{R}_{\text {or }}$, orifice index, Ur, urea.

with, however, a much higher relative incidence $(55 \%$ and $350 \%$, respectively) in the former group. Plausibly, we can suggest that these membrane defects relate to the physiological aging of RBCs that, in the HD patients, was accelerated due to a number of causes, ultimately resulting in decreased lifespan. ${ }^{5,6}$ The candidate causes can be effectively categorized into extrinsic and intrinsic classes.

- Extrinsic class: Increased mechanical stress due to kinks in the extracorporeal circulation line that can motivate macroscopic lysis of $\mathrm{RBCs}^{8,9}$ is not relevant in our case. 
However, the contribution of the roller pump or even minor mechanical stress due to the shear forces and biochemical activation due to the imperfect biocompatibility of the extracorporeal materials that unavoidably occur during dialysis cannot be neglected since these can damage RBCs. ${ }^{8-11}$ Furthermore, a number of studies have reported that both the lipid and protein compositional profile of the RBC membrane is drastically modified during dialysis. ${ }^{12-15}$ It is plausible to assume that these compositional modifications of the RBC membrane that are motivated by the extrinsic mechanisms ${ }^{12-15}$ could somehow influence the membrane structure and ultimately influence the RBC lifespan. ${ }^{5,6}$ Here we observed a negligible relative variation, $-4 \%$ and $+12 \%$ of the orifice and crevice indices, respectively, between the pre- and post-HD samples. Thus, the extrinsic mechanisms showed only minor (if any) influence on the RBCs.

- Intrinsic class: Biochemical processes between the numerous toxins of the uremic milieu and the RBCs are generally considered as the underlying cause of decreased RBC lifespan. ${ }^{16}$ In a previous work, Stamopoulos et al reported the strong correlation of the orifice index with urea, but not with creatinine. ${ }^{7}$ In the present study, excepting urea and creatinine, we investigated how the orifice index depends on the concentration of two other basic uremic markers, calcium and phosphorous. In our analysis, we divided HD patients into two subgroups, of low and high uremic marker concentration. The results were compared to those of the healthy donors. Statistically significant differences were observed between (1) the subgroups of the HD patients of low and high uremic marker concentration $(P<0.05),(2)$ the groups of HD patients and healthy donors $(P<0.01)$, and (3) the subgroup of HD patients of high uremic marker concentration and the group of healthy donors $(P<0.001)$ in the cases of urea (Figure 3A), calcium (Figure 3C), and phosphorous (Figure 3D). In the case of creatinine (Figure 3B), no statistically significant difference between the subgroups of the HD patients was observed. The respective variation of the crevice index with the concentration of uremic markers is not statistically significant due to the small absolute population of these linear defects. Statistically significant variations can probably emerge even for the crevice index when increasing the statistical sample.

Finally, we stress that, in this study, the RBCs were subjected to the minimum possible processing to preserve the native landscape of their membrane. In a recent AFM-based study, ${ }^{19}$ Stamopoulos et al demonstrated that the biochemically "inert" media that are routinely used in laboratory practice (saline, phosphate-buffered saline, etc) interact with blood cells, modifying the morphological information of the membrane, at least at the nanometer level. The same cautions were raised in a recent SEM-based study on RBCs, ${ }^{24}$ in which air-dried smears and paraffinembedded sections were compared. It was demonstrated that the size of RBCs from the paraffin-embedded sections was underestimated by approximately $30 \%$, clearly due to the over-processing employed in the method.

A limitation of the present study is that it investigated the variation of the membrane trauma (as represented by the orifice index) that possibly occurred during each 4-hour session in patients who were already on HD when the study began. Thus, we were not able to distinguish between the immediate RBC membrane trauma possibly occurring at each 4-hour session and the total trauma that possibly accumulated gradually to formerly untreated RBCs after the scheduled initiation of HD. To obtain information on this issue, end-stage renal disease patients should be investigated in a timely manner prior to initiation of HD and followed for an adequate period of time that safely exceeds the RBC lifespan, for example, for 3 months on HD treatment. The direct comparison of the results obtained prior to and after initiation of HD is important, since it will enable us to reliably distinguish between the influences of the intrinsic and extrinsic factors as follows: a decreased orifice index after initiation of HD can be rightly ascribed to the improved biochemical profile of toxins (intrinsic factor), while an increased orifice index after initiation of HD can be safely attributed to the accumulation of mechanical damage (extrinsic factor). This investigation will give additional proof to further document the results presented here. Another limitation of the present study relates to the lack of comparison between blood samples drawn at the arterial and venous lines of the dialysis machine (that is, before and after the dialysis membrane). This comparison would resolve any changes of the RBC membrane occurring while passing through the filter, possibly motivated by the rapid changes in solute concentrations and osmolarity experienced by the RBCs. Finally, no correlation was found between the orifice index and the underlying cause of end-stage renal disease for the specific HD patients studied in this work (see subsection "HD patients"), probably due to the limited number of subjects. These issues could be investigated in future works.

\section{Conclusion}

The possible detrimental action of the extracorporeal circulation to the RBC membrane was investigated with two 
powerful imaging techniques, namely, AFM and SEM. The orifice and crevice defects detected in the RBC membranes of the HD patients exhibited a negligible relative variation that was not statistically significant, $-4 \%$ and $+12 \%$, respectively, between the pre- and post-HD blood samples. Thus, extrinsic factors such as mechanical stress and/or biochemical activation cannot seriously damage the RBCs during the extracorporeal circulation. When the data of the HD patients are compared to the healthy donors, a statistically significant relative increase of order $+55 \%$ and $+350 \%$ is observed for the orifices and crevices, respectively. Interestingly, for the HD patients, the intrinsic factor, that is uremic milieu, seemed to dominate, since a correlation between the defect population and concentration of urea, calcium, and phosphorous, although not creatinine, was observed.

\section{Disclosure}

The authors report no conflicts of interest in this work.

\section{References}

1. Tomasello S. Anemia of Chronic Kidney Disease. J Pharm Pract. 2008;21:181-195.

2. Nurko S. Anemia in chronic kidney disease: causes, diagnosis, treatment. Cleve Clin J Med. 2006;73:289-297.

3. Hörl WH. Adjunctive therapy in anaemia management. Nephrol Dial Transplant. 2002;17(Suppl 5):56-59.

4. Hörl WH. Non-erythropoietin-based anaemia management in chronic kidney disease. Nephrol Dial Transplant. 2002;17 Suppl 11:35-38.

5. Ly J, Marticorena R, Donnelly S. Red blood cell survival in chronic renal failure. Am J Kidney Dis. 2004;44:715-719.

6. Vos FE, Schollum JB, Coulter CV, Doyle TC, Duffull SB, Walker RJ. Red blood cell survival in long-term dialysis patients. Am J Kidney Dis. 2011;58:591-598.

7. Stamopoulos D, Grapsa E, Manios E, Gogola V, Bakirtzi N. Defected red blood cell membranes and direct correlation with the uraemic milieu: the connection with the decreased red blood cell lifespan observed in haemodialysis patients. Nanotechnology. 2012;23:485101.

8. Hoenich N. The extracorporeal circuit: Materials, problems, and solutions. Hemodial Int. 2007;11:S26-S31.

9. Polaschegg HD. Red blood cell damage from extracorporeal circulation in hemodialysis. Semin Dial. 2009;22:524-531.

10. Cheung AK. Biocompatibility of hemodialysis membranes. J Am Soc Nephrol. 1990;1:150-161.
11. Hakim RM. Clinical implications of hemodialysis membrane biocompatibility. Kidney Int. 1993;44:484-494.

12. Dolegowska B, Kwiatkowska E, Wesolowska T, Bober J, Chlubek D, Ciechanowski K. Effect of hemodialysis on the content of fatty acids in monolayers of erythrocyte membranes in patients with chronic renal failure. Ren Fail. 2007;29:447-452.

13. Costa E, Rocha S, Rocha-Pereira P, et al. Changes in red blood cells membrane protein composition during hemodialysis procedure. Ren Fail. 2008;30:971-975.

14. Koorts AM, Viljoen M, Kruger MC. Red blood cell fatty acid profile of chronic renal failure patients receiving maintenance haemodialysis treatment. Prostaglandins Leukot Essent Fatty Acids. 2002;67:13-18.

15. Peuchant E, Salles C, Vallot C, Wone C, Jensen R. Increase of erythrocyte resistance to hemolysis and modification of membrane lipids induced by hemodialysis. Clin Chim Acta. 1988;178:271-282.

16. Kruse A, Uehlinger DE, Gotch F, Kotanko P, Levin NW. Red blood cell lifespan, erythropoiesis and hemoglobin control. Contrib Nephrol. 2008;161:247-254.

17. Müller DJ, Dufrêne YF. Atomic force microscopy as a multifunctional molecular toolbox in nanobiotechnology. Nat Nanotechnol. 2008;3: 261-269.

18. Kaasgaard T, Mouritsen OG, Jørgensen K. Lipid domain formation and ligand-receptor distribution in lipid bilayer membranes investigated by atomic force microscopy. FEBS Lett. 2002;515:29-34.

19. Stamopoulos D, Manios E, Gourni E, et al. Surveying the response of transport channels of intact rbcs membranes upon $\mathrm{AgNO}_{3}$ administration: an atomic force microscopy study. Cell Physiol Biochem. 2009;24:33-44.

20. Zachée P, Boogaerts M, Snauwaert J, Hellemans L. Imaging uremic red blood cells with the atomic force microscope. Am J Nephrol. 1994;14:197-200.

21. Stamopoulos D, Manios E, Gogola V, et al. Bare and protein-conjugated $\mathrm{Fe}(3) \mathrm{O}(4)$ ferromagnetic nanoparticles for utilization in magnetically assisted hemodialysis: biocompatibility with human blood cells. Nanotechnology. 2008;19:505101.

22. Stamopoulos D, Bouziotis P, Benaki D, Kotsovassilis C, Zirogiannis PN. Utilization of nanobiotechnology in haemodialysis: mock-dialysis experiments on homocysteine. Nephrol Dial Transplant. 2008;23:3234-3239.

23. Fassett RG, Horgan B, Gove D, Mathew TH. Scanning electron microscopy of glomerular and non glomerular red blood cells. Clin Nephrol. 1983;20:11-16.

24. Terzakis JA, Santegada E. Measurement of erythrocytes on diagnostic slides by scanning electron microscopy. Anal Quant Cytol Histol. 2000;22:244-246.

25. Swanepoel AC, Pretorius E. Scanning electron microscopy analysis of erythrocytes in thromboembolic ischemic stroke. Int J Lab Hematol. 2012;34:185-191.
International Journal of Nanomedicine

\section{Publish your work in this journal}

The International Journal of Nanomedicine is an international, peerreviewed journal focusing on the application of nanotechnology in diagnostics, therapeutics, and drug delivery systems throughout the biomedical field. This journal is indexed on PubMed Central, MedLine, CAS, SciSearch $\AA$, Current Contents ${ }^{\circledR} /$ Clinical Medicine,

\section{Dovepress}

Journal Citation Reports/Science Edition, EMBase, Scopus and the Elsevier Bibliographic databases. The manuscript management system is completely online and includes a very quick and fair peer-review system, which is all easy to use. Visit http://www.dovepress.com/ testimonials.php to read real quotes from published authors. 